\title{
Quantifying Appearance Retention in Carpets using Geometrical Local Binary Patterns
}

\author{
R. Quinones ${ }^{1,2,3} \star$, S.A. Orjuela ${ }^{1,3}$, B. Ortiz-Jaramillo ${ }^{1,2}$, L.Van Langenhove ${ }^{2}$, \\ and W. Philips ${ }^{1}$
}

${ }^{1}$ Ghent University, Department of Telecommunications and Information Processing (TELIN-IPI-IBBT)

St-Pietersnieuwstraat 41, Gent, Belgium

${ }^{2}$ Ghent University, Department of Textiles

Technologiepark 907, 9052 Gent, Belgium

3 Antonio Narino University, Faculty of Electronic and Biomedical Engineering

Cra. 58A No. 37 - 94 Bogota, Colombia

\{rquinone, seraleov, bortiz\}@telin.ugent.be

\{Lieva.VanLangenhove, Wifried.Philips\}@ugent.be

telin.ugent.be

\begin{abstract}
Quality assessment in carpet manufacturing is performed by humans who evaluate the appearance retention (AR) grade on carpet samples. To quantify the AR grades objectively, different research based on computer vision have been developed. Among them Local Binary Pattern (LBP) and its variations has shown promising results. Nevertheless, the requirements of quality assessment on a wide range of carpets have not been met yet. One of the difficulties is to distinguish between consecutive AR grades in carpets. For this, we adopt an extension of LBP called Geometrical Local Binary Patterns (GLBP) that we recently proposed. The basis of GLBP is to evaluate the grey scale differences between adjacent points defined on a path in a neighbourhood. Symmetries of the paths in the GLBPs are evaluated. The proposed technique is compared with an invariant rotational mirror based LBP technique. The results show that the GLBP technique performs better to distinguish consecutive AR grades in carpets.
\end{abstract}

Keywords: Carpet Wear Assessment, Local Binary Pattern, Texture Inspection, Image Analysis.

\section{Introduction}

Carpet manufacturers are highly interested in reducing the subjectivity in the current quality assessment method performed by human experts. Carpet quality assessment consists of quantifying the expected appearance of the carpets due to traffic exposure after a predefined time of installation. The traffic exposure is simulated by a mechanical system which accelerates the wear on samples of new

\footnotetext{
* R. Quinones is supported by Antonio Narino University
} 
carpet products $[1,2]$. Then, the quantification is assessed by evaluating surface changes following certified standards, where carpets with original appearance are graded with number 5 and carpets with severe overall change are graded with number $1[3,4]$. A set of numbers within this range defined in steps of 0.5 are assigned to grade the appearance changes. These numbers are called 'Appearance Retention' (AR) grades.

Several studies based on computer vision have been aimed to quantify the AR grades objectively [5-9]. They still are not good enough to meet the required discrimination of the AR grades imposed by standards on a sufficient wide of carpets [10]. Recently, we have proposed an automatic assessment system based on extracting texture parameters from intensity color and depth (range) images [11]. The range images are obtained using our own scanner specifically designed for carpets [12]. We have composed our own carpet database following the European standard $[3,4]$. While own earlier work should a definitive improvement of the state of the art, it still did not meet the requirements of the standard. Thus, the challenge is to perform an algorithm able to distinguish between texture features corresponding to different AR grades.

Algorithms based on Local Binary Pattern techniques have shown promising results to achieve this goal [9]. LBP techniques first describe with binary codes the changes in intensity values around a neighbourhood for each pixel in an image [13]. The binary code of each pixel is defined by thresholding the intensity values in the pixels on the neighbourhood with the intensity value of the evaluated pixel $[14,15]$. Assigning code numbers to the binary codes, the texture is statistically characterized by the probability of occurrence of the possible code numbers in the image. Grouping symmetric invariants of binary codes by using points on a circular neighbourhood improves the distinction of similar textures such as those exhibit by consecutive AR grades [9]. Also, relevant changes in appearance are better characterized by computing binary codes at different radii depending of the type of carpet [13]. Therefore, different configurations of LBP techniques evaluating neighbourhoods at different distances are necessary to describe completely a particular texture given by a certain carpet type. This results in a high feature dimension, with vectors of sizes equal to the number of neighbours points used on each circle. Many of these feature vectors have irrelevant information disturbing the discrimination between similar textures like those given by carpet surface appearance in consecutive AR grades.

We propose in this research to adopt an extension of the LBP technique, called 'Geometric Local Binary Pattern' (GLBP) to increase the distinction between consecutive AR grades. The binary codes are computed from words in points on multiple circles with different radii. A set of points defines a geometric structure. The intensity changes around a pixel are explored in a more complex neighbourhood by exploring symmetric versions of a particular structure around the pixel. Each pixel is associated with a set of binary code words representing the intensity changes on the point in the symmetric structures. The texture in an image is described statistically by the probabilities of occurrence of code numbers in the whole image or in a region of interest. 
We test the performance of the technique in distinguishing AR grades using range images of textile floor coverings from our carpet database. The GLBP technique is compared with an invariant rotational mirror based LBP technique [9] called in this paper symLBP. symLBP was our previous improvement to distinguish AR grades in carpets. The techniques are applied on four set of carpets from our database. The results show that the GLBP technique correctly distinguish more textures from consecutive AR grades than symLBP. The results are based on quantifying monotonicity, discriminance and variability from the relation between texture features and AR grades.

This paper is organized as follows: We first describe in Section 2 the carpet database to be evaluated by GLBP. Secondly, in Section 3 the proposed GLBP technique. Then, we describe in Section 4 the experiment conducted to evaluate the performance of the GLBP technique. Afterwards, results are reported in Section 5. Finally, conclusions are drawn in Section 6.

\section{Materials}

For this research, we evaluated the performance of the GLBP technique on range images of carpets to distinguish the different levels of AR grades. We used range images from our database which represent the texture on the surface of the carpets. The database is composed of images taken from physical samples of carpets following the EN1471:1996 European standard [3]. The surface of the carpets have been subjected to accelerated wear by using the Vetterman tester [16].

For this research we composed four references from the eleven types of carpet references established by the EN1471:1996 European standard. These references have been established based on a combination of characteristics in the carpet construction such as pile/surface fibre, among others. In each reference, nine degrees of surface degradation are defined. The degrees are specified by using AR grades. The AR grades vary from 1 to 5 with steps of half point, where a AR grade of 1 represents a severe change of a fatigued specimen whereas an AR grade 5 represents an original sample not exposed to any traffic exposure mechanisms.

The database contains range images of a set of carpets with different AR grades. The range images were composed by scanning the carpet samples with the scanner based on structured light described in Orjuela et al [12]. The surface reconstruction of the range image was based on a wavelet edge detector [17]. Range images represent the digitized 3D structure on the surface of the textile floor coverings. Each pixel in an image represents the depth of the surface in an area of $0.24 \mathrm{~mm}$ by $0.24 \mathrm{~mm}$. Cut-outs of of range images for transitional changes in appearance from label 1 to 5 are shown in Figure 1. 


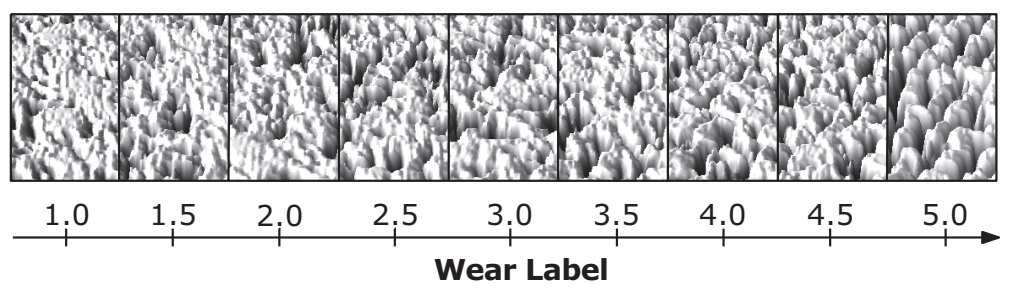

Fig. 1. Cut-outs of of range images for transitional changes in appearance from label 1 to 5 .

\section{Methods}

We defined a GLBP structure as a set of points placed on concentric circles with different radius around a evaluated pixel. The set of points are symmetrically distributed on the circles and calculated by bilinear interpolation [18]. Figure 2 a) shows the example for point on three circles with different radii into a $7 \times 7$ window.

We define $\Gamma_{(r, N)}$ as a set of $N$ points on a circle of radius $r$ from the evaluated pixel, representing one circular neighbourhood as follows: Let $p=(r, n 2 \pi / N)$ be the polar coordinates of a point $n$ in a circular neighbourhood. A circular neighbourhoods is given by the sets of points:

$$
\Gamma_{(r, N)}=\left\{p_{n}=(r, n 2 \pi / N), n=1, \ldots, N\right\}
$$

Figure 2 a) illustrates an example of a neighbourhood with points placed on three circles $\left\{\Gamma_{\left(r_{1}, N_{1}\right)}, \Gamma_{\left(r_{2}, N_{2}\right)}, \Gamma_{\left(r_{3}, N_{3}\right)}\right\}$, with corresponding radii defined by $\left\{r_{1}, r_{2}, r_{3}\right\}=\{0.707,1.93,2.97\}$ and corresponding numbers of points on each circle given by $\left\{N_{1}, N_{2}, N_{3}\right\}=\{4,8,12\}$.

In the neighbourhood, a pair of points $p_{i} \in \Gamma_{\left(r_{n}, N_{n}\right)}$ and $p_{j} \in \Gamma_{\left(r_{(n-1)}, N_{(n-1)}\right)}$ are called adjacent, if $p_{j}$ is the nearest point of the set $\Gamma_{\left(r_{(n-1)}, N_{(n-1)}\right)}$ to the point $p_{i}$. Figure $2 \mathrm{~b}$ ) shows the adjacent points for the example in Figure 2 a) The connections of the adjacent points are drawn with arrows.

Bits located on the points of the three neighbourhoods represent intensity changes in the image. The bits are calculated by thresholding the corresponding intensity of adjacent points $p_{i}$ and $p_{j}$ in the direction of the central pixel as follows:

If $I_{i}$ is the intensity value of a point located in $p_{i}$ and $I_{j}$ the intensity value of the adjacent point $p_{j}$ located in an inside circle; the bit value, termed $b_{i}$, assigned to the point $p_{i}$ is computed as follows:

$$
b_{i}=\left\{\begin{array}{l}
1, \quad\left(I_{i}>I_{j}\right) \\
0, \text { otherwise }
\end{array}\right.
$$

Figure 2 c) shows the bits obtained for the points in Figure 2 a). 


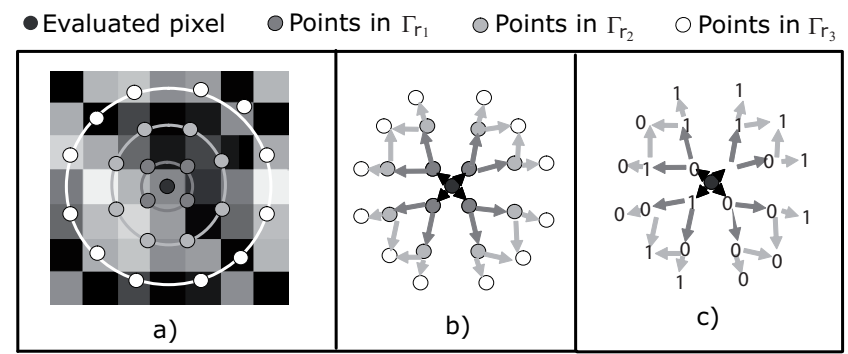

Fig. 2. Overview of the Geometric Local Binary Pattern technique. a) Points placed on three circles with different radios into a $7 \times 7$ window. The central pixel as well as the points on the different circular neighbourhoods $\Gamma_{r}$, with $r=r_{1}, r_{2}, r_{3}$, are identified with different colours b) Adjacency structure. The connections between adjacent points are drawn with arrows starting from the point that is used as threshold. c) Binary representation.

Let a path $P=\left\{p_{k}\right\}, k=1, \ldots, R$, with $R$ the number of circular neighbourhoods, be a set containing pairs of adjacent points, where each pair of points has one common point with the next pair. The path $P$ describes the primitive structure of the GLBP. Thus, the structure is completely described by a set of prototype paths under symmetry rules of rotation and complement. There are $N_{e}$ numbers of paths as the number of points in the exterior circle. Figure 2 shows the two prototype paths.

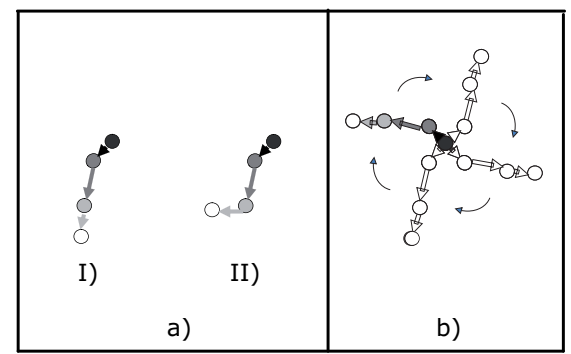

Fig. 3. a) Paths used in this approach as primitive structures of GLBP. b) Rotation of the path a)I).

If $b_{k}$ represents the bit value assigned to the point $p_{k} \in P$, the corresponding code $c$, of the intensity changes on the path is computed as follows:

$$
c=\sum_{k=1}^{R} b_{k} 2^{k}
$$


The local texture around a pixel is described with the probability of occurrence of the code numbers resulting from all detected paths around the pixel. Thus, the texture description is obtained by the union of paths describing structures called the GLBP structures. Any combination of the primitive structures results in a predefined GLBP structure with rotational and mirror symmetry invariance. The texture in an image is characterized statistically by the probabilities of occurrence of each code number $c$ computed with the bits on the points from the GLBP structure accumulated into one histogram $h(c)$. We use the Kullback-Leibler divergence to quantify the difference between the histograms $H_{1}(c)$ and $H_{2}(c)$ of the GLBPs of a non-worn sample (AR grade 5) and the worn sample (AR grade between 0 to 4.5 ). We denote $i=1,2$ the index of a histograms $H_{1}(c)$ and $H_{2}(c)$ (non-worn and worn) and $b=1, \ldots, B$ the index $b$ of a bin of a histogram $H(c)$.

With this notation, the difference between both histograms is quantified using the symmetric adaptation of the Kullback-Leibler divergence, termed $\kappa$, in eq. (4).

$$
\kappa=\sum_{i=1}^{2} \sum_{b=1}^{B} H(i, b) \log H(i, b)-\sum_{i=1}^{B} H_{p}(i) \log H_{p}(i) ; \quad H_{p}(i)=\sum_{i=1}^{2} H(i, b)
$$

One $\kappa$ value is obtained for each comparison of two textures.

\section{Experiment}

To evaluate the GLBP, we use four sets of carpets with their AR grades from range images described in section 2 based on the Europe carpet-appearance standard EN1471. The sets correspond to carpets type: loop pile (Carpet 1), cut pile (Carpet 2), Shaggy (Carpet 3) and Loop/Cut Pile (Carpet 4). The range images are evaluated by GLBP based on their $\kappa$ values. $\kappa$ values are computed comparing textures of samples with appearance changes corresponding to AR grades against samples with original appearance. Finally, we compare the performance of GLBP against an invariant rotational mirror based LBP technique (symLBP) to distinguish between consecutive AR grades. The assumptions to evaluate the performance of GLBP and symLBP for distinguishing textures related to AR grades are:

- the mean values of the texture features corresponding to AR grades change in order with respect to the AR grades.

- the texture features corresponding to consecutive AR grades are well discriminated.

These two characteristics are based on the principle of monotonicity and discriminance. Additionally, we measure the variability in the relation between the $\kappa$ values and the AR grades.

These three measures are described as follows: 
1. The monotonicity (M) evaluate the order-preserving between the extracted features and the related AR grades. This is computed by Spearman rank correlation, termed $\rho$ [19]. To compute the Spearman rank correlation, the texture features must be first ordered from small to large and then computed under the eq. (5).

$$
\rho=1-\frac{K}{(G)\left((G)^{2}-1\right)} \sum_{g=1}^{G} d_{g}{ }^{2}
$$

Where, $d_{b}$ the differences between an assigned rank and an expected rank. $g$ is the index of the $G$ number of differences $g=1, \ldots, G . K=6$, a constant defined by Spearman.

2. The discriminance (D), evaluates the efficiency in distinguishing between consecutive AR grades. The discriminance is calculated based on Tuckey Test[19] by eq. (6) and indicates whether there is a significant statistically difference in the means of the $\kappa$ values between consecutive AR grades. . Defining $F$ as the texture features, $S$ the number of texture features with $s=1, \ldots, S$, the statistical significance is computed as follows:

$$
\varsigma=\frac{q_{(\alpha, G, S G-G)}}{\sqrt{(S G-G) S}} \sqrt{\sum_{g=1}^{G} \sum_{s=1}^{S}\left(F_{g s}-\mu_{g}\right)^{2}}
$$

$q_{(\alpha, G, S G-G)}$ is obtained from the studentized range distribution at $100(1-\alpha)$ of confidence. $\mu_{g}$ is the mean value of the texture features associated with the AR grade $g$. Discriminance is finally computed as the number of times that eq. (7) is satisfied.

$$
\left(\mu_{(g+1)}-\mu_{g}\right)-\varsigma>0
$$

3. The variability ( $\mathbf{V})$, defines how well the total variation in the AR grades can be explained by the linear relation between the $\kappa$ values and the AR grades. The adjusted coefficient of determination, termed, $R_{a}^{2}$, is used [19] to quantify the variability. $R_{a}^{2}$ is defined as:

$$
R_{a}^{2}=1-\frac{n-1}{n-p-1} \frac{\sum_{i}\left(y_{i}-\hat{y}_{i}\right)^{2}}{\sum_{i}\left(y_{i}-\bar{y}\right)^{2}}
$$

Where $y_{i}$ is an AR grade computed from the features, $\hat{y}_{i}$ is the estimated AR grade assessed by humans and $\bar{y}$ is the mean of the $y_{i}$ values. $p$ is the total number of parameters in the linear model $\bar{y}=\alpha+\beta \bar{\kappa}, n$ is the number of $\kappa$ values per sample size. 


\section{$5 \quad$ Results}

We have compared the performance of the GLBP and the symLBP techniques in discriminating AR grades using samples from four carpet types of the European standard. We found that discriminance, monotonicity and variability in the description of AR grades improved in the GLBP method. The results are listed in Table 1. Table 1 shows the measurements of discriminance, monotonicity and variability obtained by evaluating symLBP and GLBP techniques for 4 carpets with their respective AR grades.

\begin{tabular}{|l|c|c|c|c|c|c|}
\hline & \multicolumn{3}{|c|}{ symLBP } & \multicolumn{3}{c|}{ GLBP } \\
\hline & $D$ & $M$ & $V$ & $D$ & $M$ & $V$ \\
\hline Carpet 1 & 6 & 0.98 & 0,80 & 7 & 1.00 & 0,89 \\
\hline Carpet 2 & 6 & 0.93 & 0,79 & 6 & 0.93 & 0,80 \\
\hline Carpet 3 & 5 & 0.98 & 0,80 & 6 & 1.00 & 0,83 \\
\hline Carpet 4 & 7 & 1.00 & 0,88 & 7 & 1.00 & 0,90 \\
\hline
\end{tabular}

Table 1. Discriminance(D), Monotonicity(M) and Variability(V) to distinguish AR grades in carpets using symLBP and GLBP.

The results show that the discriminance between consecutive pairs of AR grades for carpet types loop pile (Carpet 1: 6 to 7) and Shaggy (Carpet 3: 5 to 6 )increased using the GLBP technique, achieving a correct description of AR grades with $\kappa$ values $(M=1)$. For all carpets the variability increased (from 0.81 to 0.86 in average for the four carpets).

Figure 4 illustrates the relation between $\mathrm{AR}$ grades and $\kappa$ values for carpet type loop pile using both techniques. In Figure 4 , the $\kappa$ corresponding to AR grades are displayed with box coxes, where the center of a cox box is the mean value of the $\kappa$ values and two lines at the top and at the bottom of each cox-box represent its standard deviation.

Figure 4 shows that a correct distinction between AR grades 2.5 and 3 is obtained using the GLBP technique while the SymLBP technique confuses both AR grades.

\section{Conclusions}

A GLBP based structure applied for distinguish AR grades in carpets was presented. The techniques have been tested on four set of carpets, computing texture features using the Kullback-Leibler divergence on histograms representing the frequency of mirrored and rotational invariant patterns. We evaluated the performance of the GLBP structure and compared with symLBP for distinguishing consecutive AR grades. The results show that GLBP technique improves the 

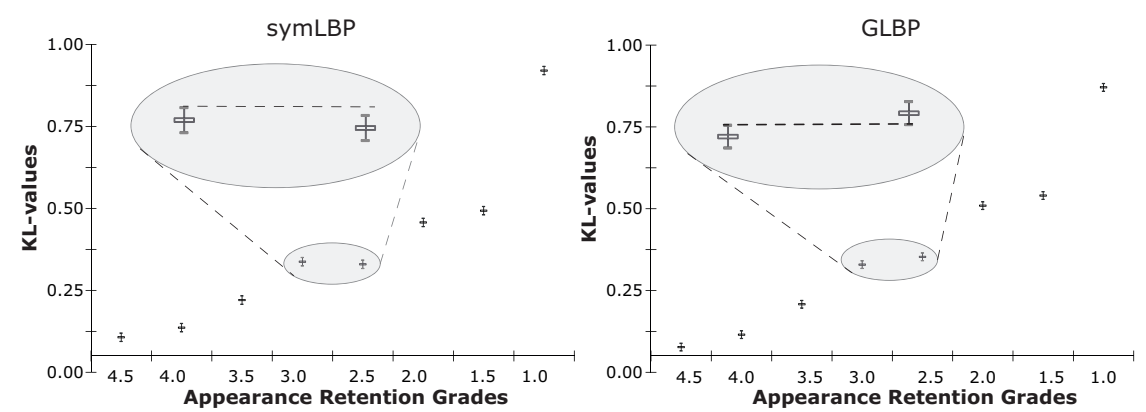

Fig. 4. Comparison between symLBP and GLBP techniques for distinguish consecutive AR grades for the loop pile carpet

performance for loop pile, shaggy and cut/loop pile carpets for distinguishing consecutive AR grades.

\section{References}

1. Textile floor coverings classification of machine-made pile rugs and runners. Textiles Floor Coverings, BS EN 14215:2003, June 2003.

2. 2010 annual book of astm standards, section 14: General methods and instrumentation, 2010.

3. Constructional details of types of textile floor covering available as reference fatigued specimens. EN1471, 1996.

4. The Carpet and Rug Institute. Cri test method - 101: Assessment of carpet surface appearance change using the cri reference scales. Technical Bulletin, 2003.

5. L. H. Siew, Hodgson R. M., and E. J. Wood. Texture measures for carpet wear assessment. IEEE Transactions on Pattern Analysis and Machine Intelligence, 10:92-105, 1988

6. E. Wood and Hofgson R. Carpet texture measurement using image analysis. Textile Research Journal, 59:1-12, 1989.

7. Y. Wu, B. Pourdeyhimi, and S.M. Spivak. Texture evaluation of carpets using image analysis. Textile Research Journal, 61:407-419, July 1991.

8. W. Waegeman, J. Cottyn, B. Wyns, L. Boullart, B. De Baets, L. Van Langenhove, and Detand J. Classifying carpets based on laser scanner data. Engineering Applications of Artificial Intelligence, 21(6):907-918, September 2008.

9. S. A. Orjuela, E. Vansteenkiste, F. Rooms, S. De Meulemeester, R. De Keyser, and W. Philips. Evaluation of the wear label description in carpets by using local binary pattern techniques. Textile Research Journal, 80(20):2132-2143, December 2010.

10. D. Van Dale and S. De Meulemeester. Annual report of department of textiles. Technical report, Ghent University, 2006.

11. S. A. Orjuela, E. Vansteenkiste, F. Rooms, S. De Meulemeester, R. De Keyser, and W. Philips. Automated wear label assessment in carpets by using local binary pattern statistics on depth and intensity images. In In Proc. of IEEE ANDESCON, pages $1-5,2010$. 
12. S. A. Orjuela, E. Vansteenkiste, F. Rooms, S. De Meulemeester, R. De Keyser, and W. Philips. Feature extraction of the wear label of carpets by using a novel 3d scanner. In Proc. of the Optics, Photonics and Digital Technologies for Multimedia Applications conference, 2010.

13. T. Ojala and Pietikainen M. A comparative study of texture measures with classification based on feature distributions. Pattern Recognition, 29(1):51-59, January 1996.

14. T. Ojala, Pietikainen M., and Menp T. Multiresolution gray scale and rotation invariant texture classification with local binary patterns. IEEE Transactions on Pattern Analysis and Machine Intelligence, 24(7):971-987, July 2002.

15. M. Ojala, T. Pietikainen and Z. Xu. Rotation-invariant texture classification using feature distributions. Pattern Recognition, 33(1):43-52, January 2000.

16. Textile floor coverings - production of changes in appearance by means of vettermann drum and hexapod tumbler testers. ISO 10361:2000, 2000.

17. S. A. Orjuela, B. Ortiz, S. J. De Meulemeester, C. Garcia, F. Rooms, A. Pizurica, and W. Philips. Surface reconstruction of wear in carpets by using a wavelet edge detector. In J. Blanc-Talon, D. Bone, W. Philips, Popescu D, and P. Scheunders, editors, In Proc. of Advanced Concepts for Intelligence Vision Systems., volume 6474, pages 309-320. Springer, December 2010.

18. S. A. Orjuela, F. Rooms, R. De Keyser, and W. Philips. Geometric local binary pattern, a new approach to analyse texture in images. In Books of Abstracts: The 2010 International Conference on Topology and its Applications, pages 179-181, June 2010.

19. M. Kutner, C. J. Nachtsheim, J. Neter, and Li W. Applied Linear Statistical Models. McGraw-Hill/Irwin, 5 edition, 2004. 